\title{
Negative differential resistance in bilayer graphene nanoribbons
}

\author{
K. M. Masum Habib, ${ }^{\text {a) }}$ Ferdows Zahid, and Roger K. Lake ${ }^{\text {b) }}$ \\ Department of Electrical Engineering, University of California, Riverside, California 92521-0204, USA
}

(Received 1 January 2011; accepted 24 April 2011; published online 13 May 2011)

\begin{abstract}
Lack of a bandgap is one of the significant challenges for application of graphene as the active element of an electronic device. A bandgap can be induced in bilayer graphene by application of a potential difference between the two layers. The simplest geometry for creating such a potential difference is two overlayed graphene nanoribbons independently contacted. Calculations, based on density functional theory and the nonequilibrium Green's function formalism, show that transmission through such a structure is a strong function of applied bias. The simulated current voltage characteristics mimic the characteristics of resonant tunneling diode featuring negative differential resistance. (C) 2011 American Institute of Physics. [doi:10.1063/1.3590772]
\end{abstract}

Graphene has fascinating electronic properties featuring the Dirac fermion ${ }^{1}$ with high mobility ${ }^{2}$ and a long coherence length. However, lack of a bandgap in two-dimensional (2D) graphene $^{3}$ reduces its utility for conventional electronic device applications. A bandgap can be introduced by patterning a $2 \mathrm{D}$ graphene sheet into a narrow $(<10 \mathrm{~nm})$ nanoribbon, known as a graphene nanoribbon (GNR). ${ }^{4,5}$ Another way to modify the band structure of graphene is to stack two monolayers to form a bilayer in which the bandgap can be tuned by creating a potential difference between the two layers. ${ }^{6,7}$

The possibility of field effect transistors (FETs) using bilayer graphene as the channel material was recently studied. ${ }^{8}$ It was shown that such a FET had a poor on-off current ratio, $I_{\mathrm{on}} / I_{\mathrm{off}}$, due to strong band-to-band tunneling. However, a tunnel FET using bilayer graphene showed promising performance. ${ }^{9}$ Other proposed devices include a nanoelectromechanical FET based on interlayer distance modulation, ${ }^{10,11}$ a FET utilizing a bilayer exciton condensate, ${ }^{12}$ and GNR junction diodes featuring negative differential resistance (NDR) based on chemical ${ }^{13}$ and field effect ${ }^{14}$ doping.

Many proposed FET type graphene based devices have multiple gates making them relatively complex device structures. We consider the simplest possible geometry by which a potential can be applied between two GNR layers. Such a geometry consists of two single layer GNRs with one placed on top of the other. Each GNR is independently contacted such that one GNR is held at ground while the other has a bias applied to it. Such a geometry and biasing scheme would occur, for example, in a cross-bar architecture. Independently contacting the top and bottom GNR maximizes the voltage drop between them. Assuming that the majority of the potential drop occurs between the two nanoribbons, the potential difference between the two nanoribbons is the applied bias. Since the bandgap increases with applied sourcedrain bias, we hypothesized that NDR would occur.

To test this hypothesis, we performed numerical simulations of a model GNR geometry using $a b$ initio density functional theory (DFT) to simulate the electronic structure and a nonequilibrium Green's Function (NEGF) approach to determine the electron transport. The model structure of the over-

\footnotetext{
a) Electronic mail: khabib@ee.ucr.edu.

${ }^{b)}$ Electronic mail: rlake@ee.ucr.edu.
}

lapping GNRs is shown in Fig. 1. It consists of a left and a right semi-infinite, armchair, H-passivated GNR which overlap in the central region. Two well known bilayer stacking sequences, $\mathrm{AB}$ and $\mathrm{AA}$, are considered. The widths of the armchair GNRs (AGNRs) are chosen to be 14 atomic C layers $(3 \mathrm{n}+2) \sim 1.8 \mathrm{~nm}$ to minimize the bandgap resulting from the finite width. The bandgap of the 14-AGNR calculated from DFT code, Fireball, ${ }^{15,16}$ is $130 \mathrm{meV}$ which is in good agreement with Son et al. ${ }^{17}$ When one GNR is stacked on top of another to form AB or AA bilayer GNRs, the bandgap is reduced further consistent with the results of Lam and Liang. ${ }^{5}$ For AA GNR, the bandgap is removed completely, and for $\mathrm{AB}$ GNR, the bandgap is reduced to $20 \mathrm{meV}$. The lengths of the overlap regions for $\mathrm{AB}$ and $\mathrm{AA}$ stacking are $1.7 \mathrm{~nm}$ and $1.6 \mathrm{~nm}$, respectively. The total simulated length between the two ideal leads indicated by the self-energies in Fig. 1 is $\sim 6.8 \mathrm{~nm}$. Transmission through similar systems in equilibrium and with gate bias was recently studied in detail with $\pi$-band and $\mathbf{k} \cdot \mathbf{p}$ models, ${ }^{18,19}$ and strong resonant and antiresonant features were observed in the transmission in agreement with our results below.

Both the AA and AB GNR bilayers are either metallic or have a bandgap less than $k_{B} T$ at room temperature. Creating a potential difference between the two layers creates a bandgap with a maximum of $0.25 \mathrm{eV}$ for the AB GNR and 130 meV for AA GNR as shown in Fig. 2. Understanding the band structure of the bilayer GNRs and the effect of bias, we are now ready to investigate the current-voltage response of the structure shown in Fig. 1. Before doing so, we provide a brief description of the theoretical models.

The electronic structures of the single and bilayer GNRs are modeled with the quantum molecular dynamics,

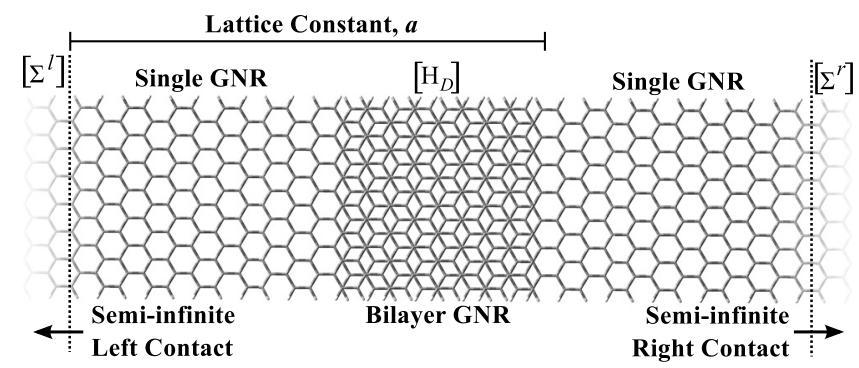

FIG. 1. Atomic geometry of modeled AB-stacked device. 


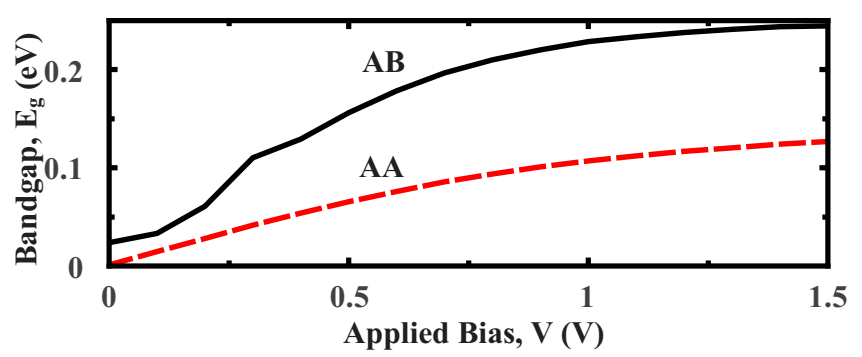

FIG. 2. (Color online) Bandgap as a function of applied bias for infinite AAand $\mathrm{AB}$-stacked bilayer GNRs.

DFT code Fireball using separable, nonlocal TroullierMartins pseudopotentials, ${ }^{20}$ the BLYP exchange correlation functional, ${ }^{21,22}$ a self-consistent generalization of the HarrisFoulkes energy functional ${ }^{23,24}$ known as DOGS after the original authors, ${ }^{25,26}$ and a minimal $s p^{3}$ Fireball basis set. The radial cutoffs of the localized pseudoatomic orbitals forming the basis are $r_{c}^{1 s}=4.10 \AA$ for hydrogen and $r_{c}^{2 s}$ $=4.4 \AA$ and $r_{c}^{2 p}=4.8 \AA$ for carbon. ${ }^{27}$

A supercell of hydrogen passivated single layer AGNR with periodic boundary conditions is relaxed quantummechanically with Fireball. The relaxed single layer supercell is then repeated to construct single-layer GNRs. These single-layer GNRs are then placed one above the other at the experimental separation distance of $3.35 \AA$ and aligned to form the $A B$ stacked structure shown in Fig. 1. The same procedure is followed to form the AA stacked structure with a separation distance of $3.55 \AA$. No further relaxation is performed on the structure. The region between the vertical lines in Fig. 1 is used as the supercell for bilayer GNR with lattice vector, a. A single point self-consistent calculation is performed with Fireball to generate the Hamiltonian matrix elements of this supercell. The matrix elements within 16 atomic layers of the end overlap regions are discarded and replaced with the matrix elements for the relaxed single-layer GNR.

The applied bias is modeled by applying a rigid shift to the energy of the lower GNR by the amount of the applied bias, $U=-e V$. The matrix elements of $U$ are calculated as $\langle i, \alpha|U| j, \beta\rangle=S_{\alpha_{i}, \beta_{j}}\left[U\left(\mathbf{r}_{i}\right)+U\left(\mathbf{r}_{j}\right)\right] / 2$ where, the indices $i$ and $j$ label the atoms, the indices $\alpha$ and $\beta$ label the basis orbitals, and $S_{\alpha_{i}, \beta_{j}}$ is the overlap matrix $\langle i, \alpha \mid j, \beta\rangle . U\left(\mathbf{r}_{i}\right)=U$ for atoms on the lower GNR and zero for atoms on the upper GNR. This approach in which the matrix elements have the same form as in an extended Huckel model has been used by others. ${ }^{28}$ The approach captures the Stark effect, but not nonequilibrium self-consistency. These and the Fireball Hamiltonian matrix elements are used in the NEGF algorithm to calculate the surface self-energies, Green's function of the device, the spectral function, the transmission, and the current as described in Ref. 29.

The simulated $I-V$ characteristics for the AB- and AAstacked GNRs corresponding to Fig. 1 are shown in Fig. 3. Both the $\mathrm{AB}$ and $\mathrm{AA}$ structure exhibit NDR. The peak current of the $\mathrm{AB}$ structure occurs at $0.4 \mathrm{~V}$ and the valley minimum occurs at $0.7 \mathrm{~V}$. The peak and valley voltages for the AA structure are approximately twice those of the AB structure. Thus, the model structure does exhibit NDR confirming the initial hypothesis.

As a check, we repeated the $I-V$ calculation of the $\mathrm{AB}$ stacked structure using a $\pi$-bond model with tight-binding

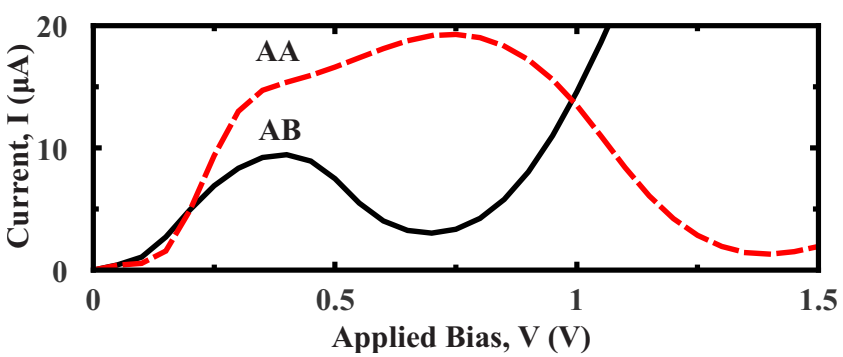

FIG. 3. (Color online) Simulated current voltage $(I-V)$ characteristics of AA- and AB-stacked devices. The valley current minimums occur at $0.7 \mathrm{~V}$ and $1.4 \mathrm{~V}$ for $\mathrm{AB}$ - and AA-stacked devices, respectively.

parameters for the intralayer coupling $(-2.569 \mathrm{eV})$ and the interlayer coupling $(-0.361 \mathrm{eV})$ taken from Ref. 30 . The peak and valley currents resulting from the $\pi$-bond model were, respectively, $24.5 \mu \mathrm{A}$ and $2.1 \mu \mathrm{A}$, occurring at the peak and valley voltages of $0.5 \mathrm{~V}$ and $1.0 \mathrm{~V}$. Thus, the two models, DFT and $\pi$-bond, give qualitatively the same $I-V$ with the $\pi$-bond model giving approximately twice the peak current and four times the peak-to-valley ratio as the DFT model.

To understand the $I-V$ characteristics shown in Fig. 3, the transmission coefficients are plotted as a function of electron energy. The transmission plots for the $A B$ structure are shown in Figs. 4(a) and 4(b) at the peak and valley bias voltages, $V=0.4 \mathrm{~V}$ and $V=0.7 \mathrm{~V}$, respectively. In both figures, the unbiased transmission and the biased quasi-Fermi levels of the left and right contacts are shown for reference.

In agreement with and as discussed in Refs. 18 and 19 the transmission shows a Fabry-Perot resonant feature at low energy and both resonances and antiresonances at more excited energies. The ends of the GNRs result in potential discontinuities at both ends of the overlap region giving rise to a resonant cavity in which multiple reflections can occur. At higher and lower energies multiple subbands allow multiple paths which can constructively or destructively interfere. Edge states also occur on the cut ends, and these states result in transmission peaks similar to those observed from the cut ends of carbon nanotubes. ${ }^{27}$

At $V=0.4 \mathrm{~V}$, the energy of the bottom GNR has been shifted down by $0.4 \mathrm{eV}$, and the low transmission regions near $E=0$ and $E=-0.4 \mathrm{eV}$ are the result of the small 130 $\mathrm{meV}$ bandgaps of the GNR leads. The region in between corresponds to transmission from hole states of the top lead to electron states of the bottom lead. As the bias of the bottom layer is increased to $0.7 \mathrm{~V}$, the dip in transmission near
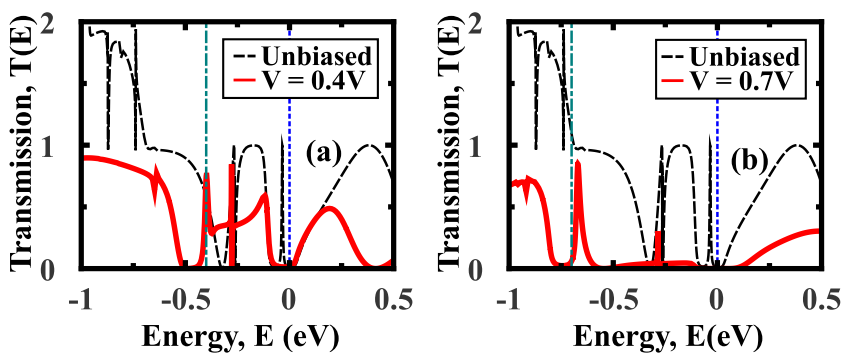

FIG. 4. (Color online) Transmission as a function of energy for AB-stacked device: (a) at bias, $V=0.4 \mathrm{~V}$ and (b) at bias, $V=0.7 \mathrm{~V}$, superimposed on transmission at no bias. The vertical lines at the lower and upper energies represent the quasi-Fermi levels of right and left contacts, respectively. The quasi-Fermi level of the left contact is set at 0 . 


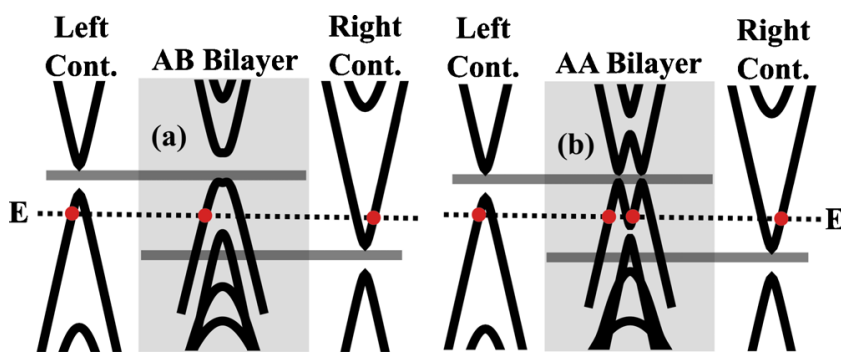

FIG. 5. (Color online) Schematic band diagrams of the leads and overlap regions of the (a) $\mathrm{AB}$ and (b) AA structures at a bias of $0.4 \mathrm{~V}$. At a given energy, $E$, the wave vectors of the leads and bilayer region are different due to the shift of bands caused by applied bias. At a given energy between the quasi-Fermi levels of the left and right contacts, the number of right moving states available for carrying current is (a) one in the $\mathrm{AB}$ device and (b) two in the AA device.

$-0.4 \mathrm{eV}$ rigidly shifts down to $\sim-0.7 \mathrm{eV}$, and the transmission from hole states to electron states between 0 and -0.7 $\mathrm{eV}$ is strongly suppressed due to the large wave vector mismatch $^{14,19}$ of the states inside the contacts and the bilayer region as illustrated in Fig. 5. The resonant feature at $\sim 0.3 \mathrm{eV}$ results from an edge state on the cut end of the top GNR.

The coherent current at any bias is proportional to the area under the transmission curve bounded by the Fermi levels of the contacts. Beyond $0.7 \mathrm{~V}$ bias, the transmission between the Fermi levels in Fig. 4(b) begins to increase as the first excited subbands of the top and bottom GNR leads are pulled into the energy window, and the current begins to increase.

The dependence of transmission of the AA device on bias follows similar trends. However, the peak current is twice as large, and the strong suppression of transmission occurs at approximately twice the bias of the $\mathrm{AB}$ device. This can be understood by noting that the wave vector mismatch for the AA case is less, since at any energy, $E$, between the quasi-Fermi levels of the left and right contacts, the AA bilayer has two states whereas the AB bilayer has only one state for right moving electrons as illustrated in Fig. 5. Therefore, more voltage is required to generate the same amount of wave vector mismatch for the AA case. This also explains the doubling of the magnitude of the peak current for the AA structure compared to that of the $\mathrm{AB}$ structure. This is consistent with the fact that there are twice as many nearest-neighbor matrix elements in AA stacking compared to those in $\mathrm{AB}$ stacking; i.e., in $\mathrm{AA}$ stacking, every atom in the lower GNR is directly below a corresponding atom in the upper GNR, whereas, in AB stacking, every second atom in the lower GNR is directly beneath an atom in the upper GNR.
In summary, we have performed ab initio DFT, $\pi$-bond, and NEGF based calculations to study the $I-V$ characteristics of a bilayer GNR structure where bias is applied between the GNRs by independently contacting each layer. The simulations of the model structures with both $\mathrm{AB}$ and $\mathrm{AA}$ stacking provide proof-of-principle that NDR can occur in such structures.

This work is supported by the Microelectronics Advanced Research Corporation Focus Center on Nano Materials (FENA).

${ }^{1}$ K. S. Novoselov, A. K. Geim, S. V. Morozov, D. Jiang, M. I. Katsnelson, I. V. Grigorieva, S. V. Dubonos, and A. A. Firsov, Nature (London) 438, 197 (2005).

${ }^{2}$ K. S. Novoselov, A. K. Geim, S. V. Morozov, D. Jiang, Y. Zhang, S. V. Dubonos, I. V. Grigorieva, and A. A. Firsov, Science 306, 666 (2004).

${ }^{3}$ T. Ando, Physica E 40, 213 (2007).

${ }^{4}$ X. Li, X. Wang, L. Zhang, S. Lee, and H. Dai, Science 319, 1229 (2008).

${ }^{5}$ K.-T. Lam and G. Liang, Appl. Phys. Lett. 92, 223106 (2008).

${ }^{6}$ H. Min, B. Sahu, S. K. Banerjee, and A. H. MacDonald, Phys. Rev. B 75, 155115 (2007).

${ }^{7}$ Y. Zhang, T.-T. Tang, C. Girit, Z. Hao, M. C. Martin, A. Zettl, M. F. Crommie, Y. R. Shen, and F. Wang, Nature (London) 459, 820 (2009).

${ }^{8}$ G. Fiori and G. Iannaccone, IEEE Electron Device Lett. 30, 261 (2009).

${ }^{9}$ G. Fiori and G. Iannaccone, IEEE Electron Device Lett. 30, 1096 (2009).

${ }^{10}$ K.-T. Lam and G. Liang, Tech. Dig. - Int. Electron Devices Meet. 2009, 37.3.1.

${ }^{11}$ K.-T. Lam, C. Lee, and G. Liang, Appl. Phys. Lett. 95, 143107 (2009).

${ }^{12}$ S. K. Banerjee, L. F. Register, E. Tutuc, D. Reddy, and A. H. MacDonald, IEEE Electron Device Lett. 30, 158 (2009).

${ }^{13}$ H. Ren, Q.-X. Li, Y. Luo, and J. Yang, Appl. Phys. Lett. 94, 173110 (2009).

${ }^{14}$ V. Nam Do and P. Dollfus, J. Appl. Phys. 107, 063705 (2010).

${ }^{15}$ O. F. Sankey and D. J. Niklewski, Phys. Rev. B 40, 3979 (1989)

${ }^{16}$ J. P. Lewis, K. R. Glaesemann, G. A. Voth, J. Fritsch, A. A. Demkov, J. Ortega, and O. F. Sankey, Phys. Rev. B 64, 195103 (2001).

${ }^{17}$ Y.-W. Son, M. L. Cohen, and S. G. Louie, Phys. Rev. Lett. 97, 216803 (2006).

${ }^{18}$ J. W. González, H. Santos, M. Pacheco, L. Chico, and L. Brey, Phys. Rev. B 81, 195406 (2010)

${ }^{19}$ J. W. González, H. Santos, E. Prada, L. Brey, and L. Chico, arXiv:1008.3255v1[cond-mat.mes-hall] (unpublished).

${ }^{20}$ J. L. Martins, N. Troullier, and S. H. Wei, Phys. Rev. B 43, 2213 (1991).

${ }^{21}$ A. D. Becke, Phys. Rev. A 38, 3098 (1988).

${ }^{22}$ C. Lee, W. Yang, and R. G. Parr, Phys. Rev. B 37, 785 (1988).

${ }^{23}$ J. Harris, Phys. Rev. B 31, 1770 (1985).

${ }^{24}$ W. M. C. Foulkes and R. Haydock, Phys. Rev. B 39, 12520 (1989).

${ }^{25}$ A. A. Demkov, J. Ortega, O. F. Sankey, and M. P. Grumbach, Phys. Rev. B 52, 1618 (1995).

${ }^{26}$ P. Jelínek, H. Wang, J. P. Lewis, O. F. Sankey, and J. Ortega, Phys. Rev. B 71, 235101 (2005).

${ }^{27}$ N. A. Bruque, M. K. Ashraf, T. R. Helander, G. J. O. Beran, and R. K. Lake, Phys. Rev. B 80, 155455 (2009).

${ }^{28}$ J. Tomfohr and O. F. Sankey, J. Chem. Phys. 120, 1542 (2004).

${ }^{29}$ N. A. Bruque, R. R. Pandey, and R. K. Lake, Phys. Rev. B 76, 205322 (2007)

${ }^{30}$ C. L. Lu, C. P. Chang, Y. C. Huang, J. M. Lu, C. C. Hwang, and M. F. Lin, J. Phys.: Condens. Matter 18, 5849 (2006). 